\title{
【Transaction】
}

\section{Changes in the Higher-Order Structure of Silk Fibers Obtained by Forcibly Spinning as Compared to Those Obtained by the Naturally Spinning System of the Bombyx mori Silkworm}

\author{
Yutaka Kawahara*1.\#, Taiyo Yoshioka*2.\#, and Kohji Tashiro*3 \\ ${ }^{* 1}$ Division of Environmental Engineering Science, Gunma University, 1-5-1, Tenjin-cho, Kiryu 376-8515, Japan \\ ${ }^{* 2}$ Silk Materials Research Unit, National Agriculture and Food Research Organization (NARO), 1-2 Ohwashi, \\ Tsukuba, Ibaraki 305-8634, Japan \\ ${ }^{* 3}$ Department of Future Industry-oriented Basic Science and Materials, Toyota Technological Institute, 2-12-1 Hisakata, \\ Tempaku-ku, Nagoya 468-8511, Japan
}

\begin{abstract}
Forcibly spinning was applied to fully grown Bombyx mori silkworm larvae, and the higher-order structure of the as-spun filaments was analyzed by Fourier-transform infrared spectroscopy (FT-IR) and wideangle X-ray diffraction (WAXD). The FT-IR spectra of the as-spun filaments were similar to those of naturally spun cocoon filaments. This indicated that the forcibly spun filaments were comprised of $\beta$-sheets. However, FT-IR spectra were obtained under ATR mode. Thus, the spectra basically reflected the structure of sericin layer as well as inside fibroin. In the WAXD diagram of forcibly spun filaments, 002 and 101 reflections of the silk-1 crystal were overlapped on the silk-2 crystal reflections, while only silk-2 crystal modification occurred in naturally spun ones. The preferential silk-2 crystal modification was disordered although the shear force loaded by the forcibly spinning on the liquid silk in the duct of a silkworm was thought to be high enough to allow modification for the preferred silk-2 crystal. In addition, the sericin layer of the forcibly spun filaments was so brittle that numerous cracks were generated perpendicular to the fiber axis when the filaments were slightly stretched, suggesting that molecular orientation in the sericin layer of the forcibly spun filaments was extremely low. These structural changes observed in the forcibly spun filaments are due to the interference of forcibly spinning with the naturally spinning system of a Bombyx mori silkworm. For further understanding the inherent spinning system of a Bombyx mori silkworm, the schematic representation was proposed based on the previous studies, and the structural changes observed in the forcibly spun filaments were discussed in relation to the fiber formation process. (Received 10 September, 2018; Accepted 30 November, 2018)
\end{abstract}

\section{Introduction}

The mechanical spinning of liquid dragline silk discharged from the spinneret of a spider is known as "forcibly spinning" [1]. The tensile strength of forcibly spun dragline silk filaments obtained from European garden spiders (A. disdematus) was enhanced by $10 \%$ when the forcibly spinning speed was increased to $100 \mathrm{~mm} / \mathrm{s}$, which is much higher than the speed of naturally spinning [1]. In the follow, the optimal forcibly spinning conditions were investigated, and the effectiveness of forcibly spinning was confirmed $[2,3]$.

This spinning technique was proved also to achieve Bombyx mori silkworm filaments with a tensile strength comparable to that of spider dragline silk fibers [4]. In recent time, extensive investigations were thus made to determine the most efficient spinning conditions for silk from the silkworm [5-7]. However, to achieve stable forcibly spinning of liquid silk discharged from the spinneret of Bombyx mori silkworm by stopping the movement of the head in arcs appears highly critical to obtain a continuous filament longer than $500 \mathrm{~m}$. Therefore, a customsynthesized paralytic peptide was used [7]. Unfortunately, the tensile strength of the forcibly spun fibers obtained with paralyzed silkworms was approximately half that of the naturally spun fibers. This marked decrease in tensile strength shows the interference of forcibly spinning with the naturally

\# corresponding author: Yutaka Kawahara (E-mail: kawahara@gunma-u.ac.jp, Tel/Fax: +81-277-30-1491) 
spinning system of silkworms.

In a case of Nephila spider, the liquid silk making up dragline silk also shows a core-shell type morphology with the interface between core main proteins called spidroin I, II, and outer coat protein. They are synthesized in the major ampullate gland and can form liquid crystalline structures with miscibility each other [8]. Thus, momentary large deformation can be applied to the liquid silk of Nephila spider. On the contrary, the liquid crystalline properties have never been confirmed in Bombyx mori sericin. Thus, cohesive failure will occur in the sericin layer of Bombyx mori filament when large deformation applied suddenly, which dominates the forcibly spinning speed. Therefore, a remarkable difference in the spinnability between Nephila spider silk and Bombyx mori silkworm silk could be observed.

Recently, the functionality of each sericin fraction, i.e. P, M, A, of Bombyx mori silkworm in relation to the fiber formation was investigated [9]. The sericin M fraction was found to work as a crystallization inhibitor against silk-2 crystal modification of fibroin, and the preferential silk-2 crystal modification of fibroin H-chains at room temperature could be guided by sericin $\mathrm{P}$ fraction like a nucleation agent [9]. Further, a new protein fraction in liquid silk, with the function of possibly promoting the solation of fibroin gel, was isolated [10]. These results suggest that the spinning system of Bombyx mori silkworm is more highly sophisticated, and therefore, the forcibly spinning should be modified to a tailor-made one when applied to Bombyx mori silkworms.

In this study, the structural changes in Bombyx mori silkworm filaments obtained by forcibly spinning were investigated in relation to the naturally spinning system of the silkworm and the naturally spun filaments, and then the schematic representation of the spinning system of Bombyx mori silkworm was proposed and discussed. Discussions on an applicability of the forcibly spinning to Antheraea wild silkworms were also made comparing the differences in physical properties between Bombyx mori silkworm silk and Antheraea wild silkworm silk.

\section{Experimental}

\subsection{Silkworm}

Fully grown domestic Bombyx mori silkworm larvae, a single cross-breed Gunma×200, were kindly supplied by the Gunma Sericultural Technology
Center.

\subsection{Forcibly spinning}

Fig. 1 shows the typical experimental conditions for forcibly spinning. For short-time spinning, generally a silkworm is grasped with fingers. The winding speed of the roller was maintained at $28 \mathrm{~mm} / \mathrm{s}$ by an electric motor device.

\subsection{Dyeing}

The sericin layer of the as-spun filaments was selectively dyed in a bath containing C.I. Direct Blue 71 (Sirius Supra Blue BRR) with a concentration of $2 \mathrm{~g}$ $/ \mathrm{L}$ at room temperature. The as-spun filaments were immersed for 1 min (liquor-to-fiber ratio of ca. 1000:1), then rinsed with water and dried in air.

\subsection{Measurements}

Fourier-transform infrared spectroscopy (FT-IR) spectra were obtained with an FT/IR 6600 spectrometer (JASCO Co., Hachioji, Japan) in diamond attenuated total reflection (ATR) mode with a TGS crystal. The spectra were generated from an average of 16 scans at a $4 \mathrm{~cm}^{-1}$ resolution.

The samples used for X-ray diffraction were prepared by forming the fibers into a bundle. By adjusting the mass of the sample, the volume of the sample irradiated by the X-rays was kept approximately constant. The crystal modification of the specimen fibers was investigated by WAXD analysis with an R-Axis Rapid II X-ray diffractometer (50 kV, 100 mA, MoK ${ }_{\alpha}$ (Rigaku Co., Japan) equipped with a cylindrical imaging plate camera.

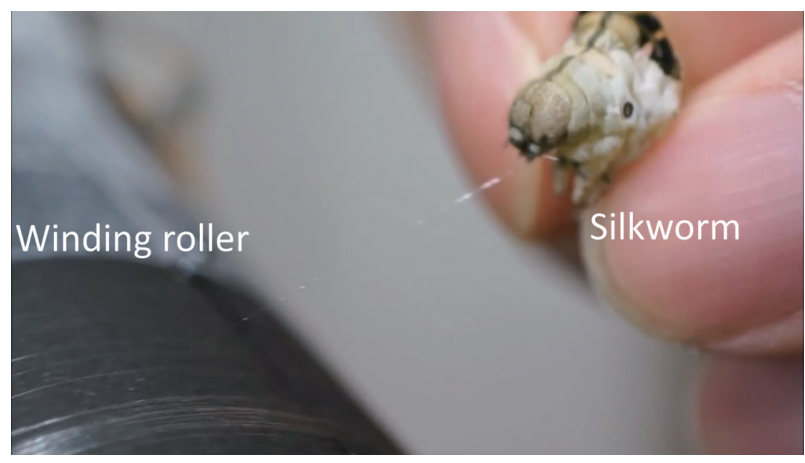

Fig. 1 Typical conditions for the forcibly spinning experiments.

\section{Results and discussion}

\subsection{Higher-order structure}

Fig. 2 shows the morphology of the as-forcibly spun silk fibroin filaments after being dyed with C.I. Direct Blue 71. The irregular thickness of the sericin layer can be clearly seen in Fig. 2 (a). Such an 


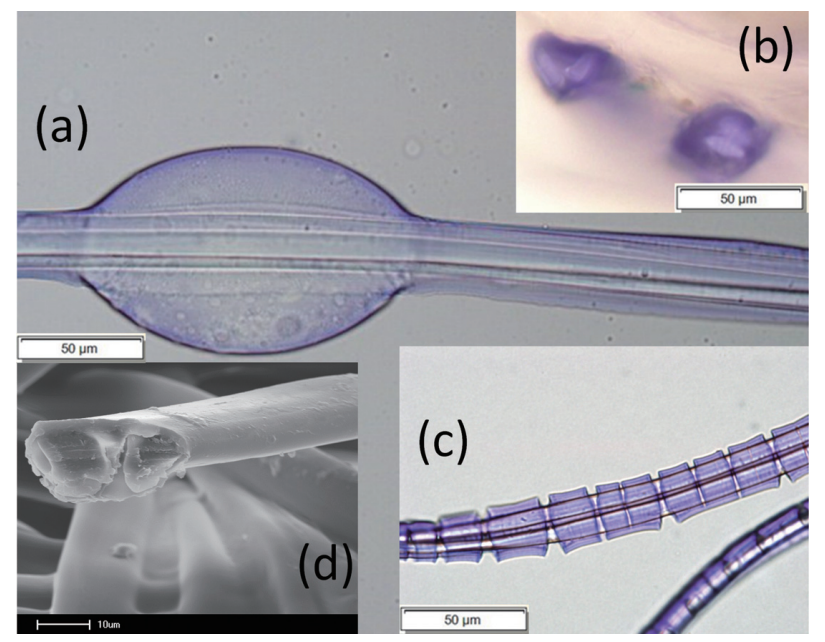

Fig. 2 Aspects of the as-spun filaments: forcibly spun filaments after being dyed with C.I. Direct Blue 71, (a) longitudinal; (b) cross-sectional; (c) after being slightly stretched (each scale bar corresponds to $50 \mu \mathrm{m}$ ), and (d) naturally spun cocoon filaments obtained from the silkworm of the same breed (the scale bar corresponds to $10 \mu \mathrm{m})$.

irregular deposition of sericin on the filament suggests that the fiber formation process in the duct of the silkworm accompanying so-called "drawdown" phenomenon is not uniform [11]. The "drawdown" point in the duct corresponds to the onset of solidification of the liquid silk although the fresh silk filament is swollen with water. In the duct, the silk press, a special valve controlled by the muscles of the silkworm; regulates the thickness of the filament and squeezes the sericin layer to smoothen the filament surface. The irregular deposition of sericin suggests that forcibly spinning may interfere with the functioning of the silk press, which is designed to maintain a spinning speed in the range of approximately $3.1 \mathrm{~mm} / \mathrm{s}\left(20^{\circ} \mathrm{C}\right)$ to $8.4 \mathrm{~mm} / \mathrm{s}\left(30^{\circ} \mathrm{C}\right)$ [12]. Therefore, it appeared that a forcibly spinning speed of $28 \mathrm{~mm} / \mathrm{s}$ was too high for the silk press system of silkworms to be able to regulate fiber formation. Furthermore, the cross-section of the fibroin filament (see Fig. 2 (b)) was ribbon-like, in contrast to the triangular shape of the naturally spun fibroin filaments of the cocoon with smooth surface (see Fig. 2(d), a twin triangular cross-section of fibroin filament could be seen in the cross-section of the cocoon thread). In addition, when the dyed as-spun filament was slightly stretched, numerous cracks were generated on the surface perpendicular to the fiber axis (see Fig. 2 (c)). The brittleness of the sericin surface layer suggests its lower molecular orientation, because even amorphous sericin could be used as a starting material for the high performance films with tensile strength over $100 \mathrm{MPa}$ as far as the drawing of molecular chains were fully made [13,14]. Moreover, a peculiar phenomenon observed at tensile behavior of forcibly spun silk filament was the generation of definite yielding although such phenomenon has never been observed for naturally spun Bombyx mori silk filament [7,15]. Therefore, it is likely that the forcibly spinning interfered with the motion of the silk press in the duct.

To investigate the higher-order structure of the forcibly spun filaments, FT-IR spectra were recorded and compared with those of naturally spun cocoon filaments (see Fig. 3). In the spectra of the forcibly spun filaments, the peaks in the amide I, II, III bands appeared at 1617,1515 , and $1235 \mathrm{~cm}^{-1}$, similar to the spectra of naturally spun cocoon filaments, with a difference of less than $1 \mathrm{~cm}^{-1}$. This indicates that the forcibly spun filaments were predominantly comprised of $\beta$-sheets [16]. However, FT-IR spectra were obtained under ATR mode. Thus, it should be noted that the spectra basically reflect the structure until several micrometers from the surface of filament [17]. Considering the thickness of sericin coating (see Fig. 2(d)), FT-IR spectra have reflected the structure of sericin layer as well as inside fibroin.

Fig. 4 shows the WAXD diagram, with its equatorial and meridional intensity curves, of a bundle of forcibly spun filaments. A well-oriented crystalline

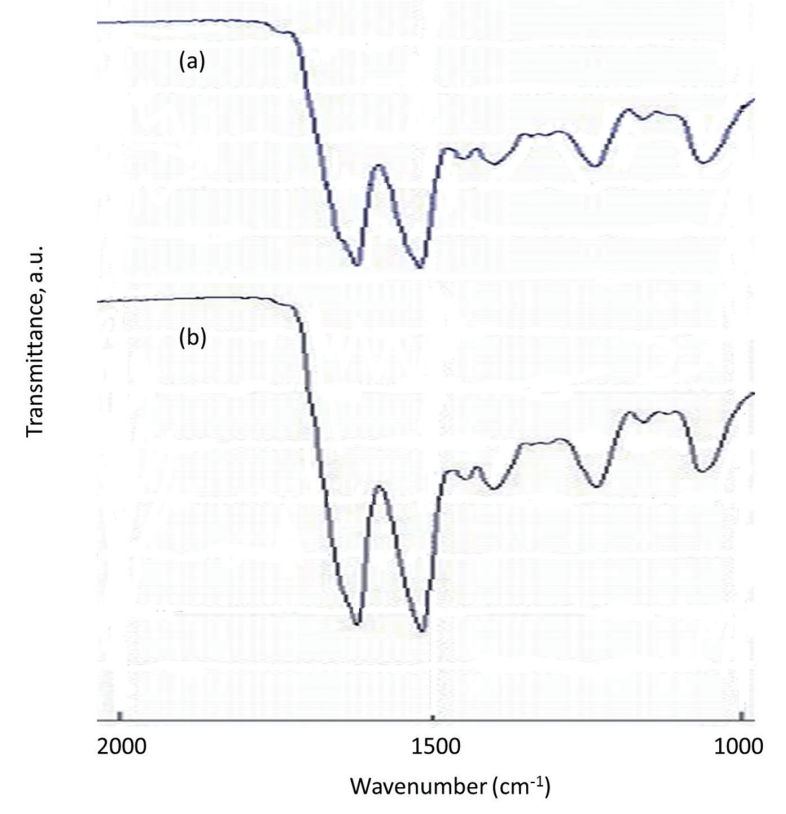

Fig. 3 FT-IR spectra for the raw silk filaments: (a) forcibly spun filaments; (b) naturally spun cocoon filaments obtained from the silkworm of the same breed. 

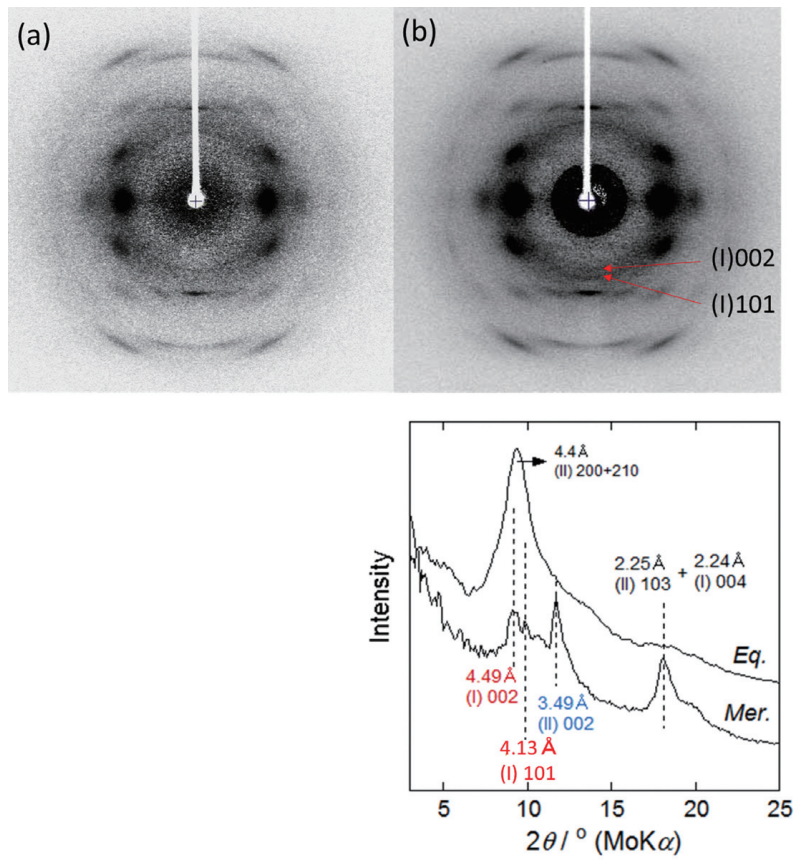

Fig. 4 WAXD fiber diagram: (a) naturally spun cocoon filaments obtained from the silkworm of the same breed; (b) forcibly spun filaments obtained at a winding speed of $28 \mathrm{~mm} / \mathrm{s}$ with its equatorial and meridional intensity curves. The fiber axis is vertical.

fiber diagram indicated that the orientation-induced crystallization proceeded almost completely. Surprisingly, however, some reflections, assigned to the silk-1 crystal modification [18], were overlapped on the meridian, indicating that the preferential silk-2 crystal modification [9] normally obtained by naturally spinning could be interrupted by forcibly spinning. Nevertheless, sericin can crystallize, and its crystal reflects strongly at Bragg's angles corresponding to $d$-spacings of 4.58, 3.68, and $3.36 \AA$ [19]. However, the reflections from sericin could hardly be seen in the
WAXD curves shown in Fig. 4, suggesting that the sericin layer covering the fibroin filaments was amorphous.

\subsection{Spinning system of the Bombyx mori silkworm}

Cocooning is basically regulated by the homeostatic metabolic system of silkworms. Therefore, applying additional force to the naturally spinning process, assuming that a silkworm is just a machine extruding liquid silk, or developing a genetically modified silkworm that produces modified fibroin, leaving the naturally spinning system of a silkworm unmodified, are not effective ways of improving the tensile strength of the silk fibers. Such strategies will merely interfere with the naturally spinning system of the silkworm, which is designed for naturally cocooning using its original liquid silk [9,20]. Fig. 5 shows a schematic representation for the spinning system of Bombyx mori silkworm according to our previous works $[9,10]$.

The silk gland is divided into three divisions: posterior (p), middle (m), and anterior (a). Fibroin is synthesized by the secretory cells in the $\mathrm{p}$ division, whereas sericin is secreted in the $\mathrm{m}$ division. The posterior division of the silk gland is narrow and approximately $15 \mathrm{~cm}$ long, is composed of 500 secretory cells, and remains curved between the dermo-visceral muscle and the trachea. The middle division is approximately $7 \mathrm{~cm}$ long and is composed of 350 secretory cells. Finally, the anterior division has no secretory cells [21]. Thus, the silk gland is equipped with a long tapering tubular (a) division after the two protein production divisions of $(\mathrm{p})$ and $(\mathrm{m})$ divisions.

The middle division of the silk gland is further divided into four parts on the basis of the different

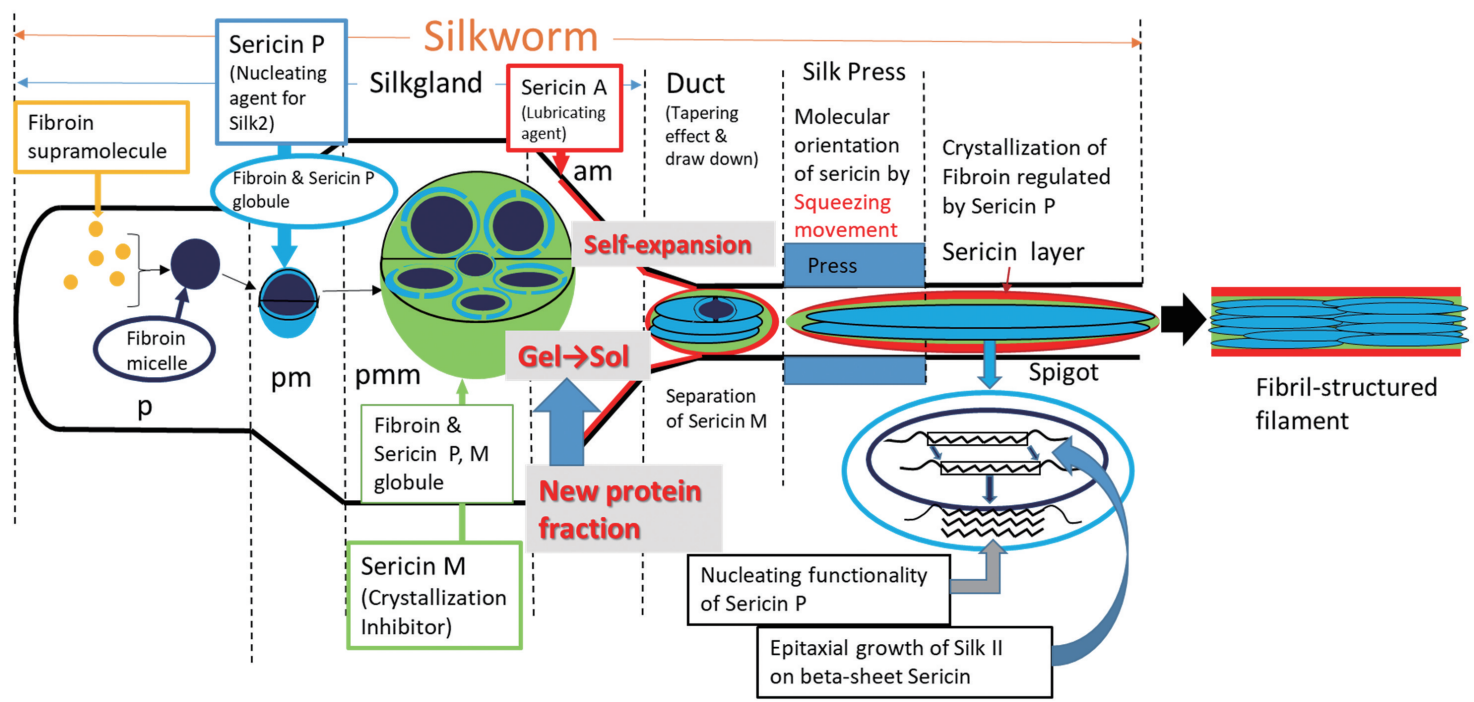

Fig. 5 Schematic representation of the spinning system of a Bombyx mori silkworm. 
functions of the secreted sericin fractions: posterior division of the middle (pm), posterior part of the middle division of the middle (pmm), anterior part of the middle division of the middle (amm), and anterior division of the middle (am) (see Fig. 5). Sericin consists of three fractions designated as $\mathrm{P}, \mathrm{M}$, and $\mathrm{A}$. Sericin $\mathrm{P}$ is secreted by the secretory cells in the pm part, sericin $\mathrm{M}$ is secreted in the amm and pmm parts, and sericin A is secreted in the amm and am parts [22].

Fibroin synthesized by the secretory cells of the (p) division is secreted into the lumen of the silk gland filled with water. To be able to be dispersed in water, fibroin H-chains containing alternate and repeated hydrophobic and hydrophilic amino sequences have to adopt a modified morphology. The stable dispersion of fibroin H-chains is made possible by the formation of micelle assemblies based on the hydrophilichydrophobic copolymeric structure, with larger terminal hydrophilic parts in contact with the surrounding water [23]. The presence of small spherical droplets in liquid silk, previously confirmed by transmission electron microscopy observations [24], is indicative of the aggregation of fibroin into micelles. In contrast, spidroin chains synthesized by a Nephila spider disperse in water through the formation of hexagonal columnar packed structures instead of micelle assemblies [8], which suggests clear differences in the fiber formation process of the Bombyx mori silkworm and the Nephila spider.

Fibroin micelles move forward, in the course of the dispersion process, into the wider middle division, where sericin fractions are secreted. The first contact of the fibroin micelle with sericin $\mathrm{P}$ fractions seems to be effective in promoting the silk-2 crystal modification in fibroin, as confirmed by the WAXD of freeze-dried specimens obtained from the pm part of the silk gland. Thus, it has been suggested that sericin $\mathrm{P}$ fractions could work as a nucleation agent that promotes the silk-2 crystal modification [9]. The contact of fibroin micelles with sericin $\mathrm{P}$ fractions tends to yield globular structures, where sericin $\mathrm{P}$ covers the inner fibroin based on the phase-separation phenomenon observed in vitro between fibroin and sericin [25], and the hydrophilicity of sericin [19].

Furthermore, at the next stage, where sericin M fractions are secreted, the occurrence of silk- 1 crystal modification was confirmed, indicating that sericin $\mathrm{M}$ fractions may regulate crystal modifications in fibroin and inhibit silk-2 crystal modification [9]. To regulate crystal modifications, sericin $\mathrm{M}$ fractions interfere with the synergic relationship between fibroin and sericin $\mathrm{P}$. In addition, sericin $\mathrm{M}$ forms a globular structure containing several fibroin and sericin $\mathrm{P}$ globules, leading to the formation of a fibrillar structure when elongated by the tapering effect of the long spinning duct and the squeezing motion at the silk press.

The third sericin fraction, sericin A, is thought to have little impact on the fiber formation process. The function of sericin $\mathrm{A}$ is to reduce friction between the spinneret surface and the inner liquid silk [26,27]. This has been confirmed by polarizing microscopic observations of the spinning duct, which indicated a very thin layer of sericin flowing along the spinneret surface [11]. The sericin layer, however, observed by polarizing microscopy was too thin to satisfy the mass balance of fibroin and sericin in liquid silk. Thus, it is likely that this thin layer was sericin A secreted into the lumen at the final parts of the middle division of the silk gland, i.e., the amm and am parts

For the Nephila spider [8], when the molecular orientation of liquid silk exceeds a certain level because of the tapering effect in the spinning duct, the "drawdown" phenomenon takes place so that the silk filament is formed although it is still swollen with water. In contrast, for the Bombyx mori silkworm [11], just before the drawdown process, the anisotropic texture of liquid silk becomes isotropic. This is quite different from what occurs in the spinning system of the Nephila spider [8]. Furthermore, just after the drawdown process, the silk press squeezes the fresh filament, which is essential to obtain a filament with a smooth surface. This essential peculiar process may be attributed to the non-liquid crystalline properties of sericin. By contrast, the two spidroin and coating proteins of Nephila spider [8] exhibit liquid crystalline behavior, and this is why forcibly spinning is directly applicable to Nephila spider silk. That means, the isotropic texture observed just before the drawdown process of the Bombyx mori silkworm silk is a sign of the structural changes occurring in liquid silk such as the disentanglement of sericin $\mathrm{M}$ from fibroin chains. The dissociation of the sericin $\mathrm{M}$ fraction from fibroin is possibly achieved through the shear force generated within the complex flow in the duct, then the sericin $\mathrm{P}$ promotes the preferred silk- 2 crystal modification in fibroin regardless of the spinning habits of the silkworm. The tensile strength of naturally spun commercial cocoon filaments amounts to around $4 \mathrm{gf} /$ denier (corresponding to $484 \mathrm{MPa}$ 
when converted using the density of $1.373 \mathrm{~g} / \mathrm{cm}^{3}$ of raw silk filaments containing $25 \%$ sericin [28]) irrespective of the body sizes and spinning habits of the silkworms [29].

As for the solation of liquid silk it has been proposed that changes in the ionic balance between $\mathrm{Ca}$ and $\mathrm{K}$ can effectively control the gelation and solation of the liquid silk [30-32]. It has been suggested that sericin can also act as a water-absorbing resin that removes water from fibroin, thus increasing its local concentration [23]. Recently, a new protein fraction in the liquid silk was isolated showing the function of possibly promoting the solation of the fibroin gel [10]. Accordingly, it seems difficult to attribute the changes in the ionic balance between $\mathrm{Ca}$ and $\mathrm{K}$ to the formation of a higher-order structure in silk fibers directly.

It has been suggested that the head movement of silkworms in figure-of-eight arcs serves to extract the liquid silk from the silk gland. However, once spinning starts, a silkworm cannot stop discharging liquid silk, not even when the movement of its head is artificially stopped [33]. Moreover, on the basis of the previously reported self-uniaxial expansion property of the liquid silk gel in water [9], it is likely that this functionality may serve to discharge liquid silk by raising the internal pressure in the silk gland effective in maintaining physical exertion of the silkworm which allows it to continue spinning for approximately 2 days without resting [12].

\subsection{Forcibly spinning of wild silkworms}

To establish a stable system for the production of

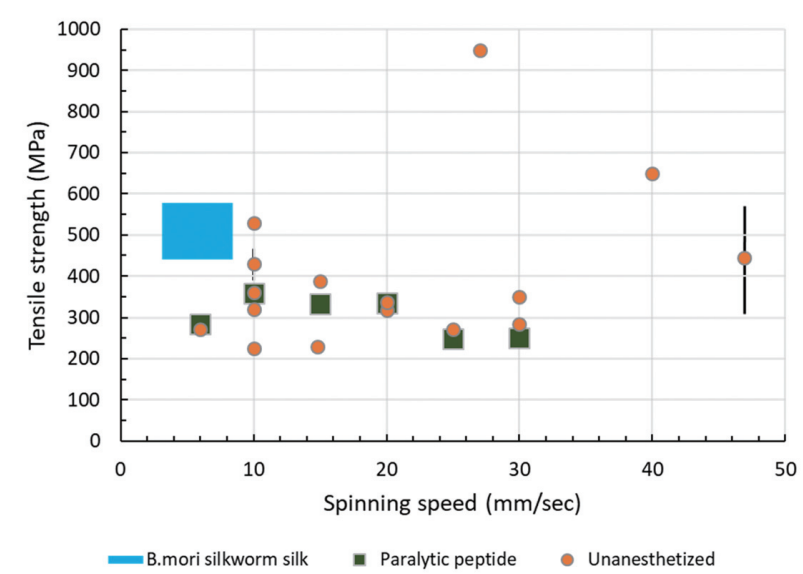

Fig. 6 Dependence of tensile strength of forcibly spun filament on the spinning speed using a silkworm anesthetized with paralytic peptide (green square), unanesthetized (orange circle). The blue area corresponds to the data for naturally spun silk fibers. silk filaments by forcibly spinning, the silkworm needs to be anesthetized so as to cease its head movement in arcs, thus preventing breaking of the filaments and affording long filaments with a length comparable to that of commercial cocoon filaments. For this purpose, a paralytic peptide was customsynthesized [7]. However, the spinning system of the Bombyx mori silkworm works via rather sophisticated mechanisms as discussed in the previous section. Therefore, applying forcibly spinning to a Bombyx mori silkworm offers very little chance of success for enhancing the tensile properties of silk filaments. Previous works on the forcibly spinning are summarized in Fig. 6 from the viewpoint of the relation between the spinning speeds and the tensile strengths of filaments obtained [4-7,15,29]. It is seen that most of the forcibly spun filaments could not exceed the range of strengths for naturally spun ones. Therefore, forcibly spinning may be inappropriate in enhancing the tensile strength of Bombyx mori silkworm silk filaments and may only be applicable when producing thinner filaments [20].

In case of Nephila spider, the animal is usually anesthetized with carbon dioxide gas [1]. The reason why carbon dioxide can be used to anesthetize a Nephila spider, but not a Bombyx mori silkworm, is because the adsorption of carbon dioxide gas by the silkworm is much greater than that by the spider [34]. As is however known, too high an adsorption of carbon dioxide can cause toxicity. However, carbon dioxide gas may be used to anesthetize several kinds of wild silkworms because the adsorbed amount is slightly smaller than that for a Nephila spider [34]. As for anesthetizing a Bombyx mori silkworm, a suffocation treatment by soaking the silkworm in room-temperature water was found effective in keeping the tensile strength of its forcibly spun silk filaments comparable to that of naturally spun silks. The detail will be published elsewhere.

A special situation as to the potential of fibers to become improved in mechanical strength by forcibly spinning exists with Antheraea pernyi silk. The amount of sericin contained in Antheraea pernyi silk is about one half that contained in Bombyx mori silk [35], which will be advantageous in terms of reaching a higher forcibly spinning speed, i.e. enhancing the spinnability of liquid silk. Furthermore, Bombyx mori silk is prone to two crystal modifications [18,36-38], whereas only one is observed in Antheraea silkworm silk [39,40]. This latter reduces the occurrence of defects due to crystal 
polymorphism in forcibly spun filaments. Moreover, reactive pigments cross-linking adjacent amorphous chains in Antheraea silk [41-43] may effectively strengthen forcibly spun filaments. Actually, the subsequent drawing of Antheraea pernyi silkworm silk in room temperature water increases the tensile strength up to $742 \mathrm{MPa}$ [44], compared to the average tensile strength of industrially reared Bombyx mori silkworm silk of $484 \mathrm{MPa}$ [29].

\section{Conclusions}

Forcibly spinning was applied to fully grown Bombyx mori silkworm larvae, and the higher-order structure of the as-spun filaments was investigated. The generation of cracks in the sericin layer covering the as-spun filament when the filaments were slightly stretched suggested a lower molecular orientation in the sericin layer. Furthermore, the preferred silk-2 crystal modification was disrupted even though the shear force was considered high enough for this modification to take place. Thus, forcibly spinning tends to interfere with the naturally spinning mechanism of the Bombyx mori silkworm. Therefore, it is to be concluded that forcibly spinning may be inappropriate in enhancing the tensile strength of Bombyx mori silkworm silk filaments in general, but may be eventually applicable to very thin filaments.

\section{Acknowledgments}

The authors thank Mr. Nobuo Kuwabara of the Gunma Sericultural Technology Center for supplying mulberry silkworms, also Mr. Yoshimitsu Ikeda of the Tokyo Metropolitan Industrial Technology Research Institute for the dyeing and optical microscopic observations.

\section{References}

1. F. Vollrath, B. Madsen, and Z. Shao, Proc. R. Soc. Lond. B, 268, 2339 (2001).

2. J.P.-Rigueiro, M. Elices, G. R. Plaza, J. I. Real, and G. V. Guinea, J. Experimental Biol., 208, 2633 (2005).

3. J. P.-Rigueiro, M. Elices, G. R. Plaza, J. I. Real, and G. V. Guinea, J. Experimental Biol., 209, 320 (2006).

4. Z. Shao, and F. Vollrath, Nature, 418, 741 (2002).

5. J. P.-Rigueiro, M. Elices, J. Llorca, and C. Viney, J. Appl. Polym. Sci., 82, 1928 (2001).

6. Md.M.R. Khan, H. Morikawa, Y. Gotoh, M. Miura,
Z. Ming, Y. Sato, and M. Iwasa, Int. J. Biol. Macromol., 42, 264 (2008).

7. B. Mortimer, C. Holland, and F. Vollrath, Biomacromolecules, 14, 3653 (2013).

8. F. Vollrath, and D.P. Knight, Nature, 410, 541 (2001).

9. Y. Kawahara, T. Yoshioka, H. Minami, H. Wakizaka, K. Tashiro, and Y. Nishikawa, J. Fiber Sci. Technol., 74, 95 (2018).

10. Y. Kawahara, S. Okamura, and H. Wakizaka, Nippon Silk Gakkaishi, 26, 13 (2018).

11. T. Asakura, K. Umemura, Y. Nakazawa, H. Hirose, J. Higham, and D. Knight, Biomacromolecules, 8, 175 (2007).

12. T. Yamamoto, M. Enokijima, I. Kamijyou, M. Fukasawa, M. Maruyama, K. Nakayama, and Y. Mimura, Bull. Sericul. Exp. Sta., 134, 81 (1988).

13. H. Teramoto, and M. Miyazawa, Biomacromolecules, 6, 2049 (2005).

14. H. Teramoto, A. Kakazu, K. Yamauchi, and T. Asakura, Macromolecules, 40, 1562 (2007).

15. Y. Kawahara, S. Kitajima, and C. Shimaoka, Journal of Textile Engineering, 64, 141 (2018).

16. X. Chen, Z. Shaoa, N.S. Marinkovic, L.M. Miller, P. Zhou, and M.R. Chance, Biophys. Chem., 89, 25 (2001).

17. R. Iwamoto, Kobunshi, 38, 906 (1989).

18. T. Asakura, K. Ohgo, K. Komatsu, M. Kanenari, and K. Okuyama, Macromolecules, 38, 7397 (2005).

19. K. Komatsu, Chemical and structural characteristics of silk sericin. In "ZokuKenshinokozo.” (N. Hojo Ed.), Shinshu Univ., Ueda, pp. 379-415 (1980).

20. Y. Kawahara, and T. Hananouchi, J. Natural Fibers, (2018). DOI:10.1080/15440478.2018.1470059

21. M. Shamsuddin, "Silkworm physiology," Daya Publishing House, New Delhi, pp. 37-43 (2018).

22. A. Shibukawa, Bull. Sericul. Exp. Sta., 15, 383 (1959).

23. H.-J.-Jin, and D.L. Kaplan, Nature, 424, 1057 (2003).

24. H. Akai, Experientia, 39, 443 (1983).

25. H. W. Kwak, J. E. Ju, M. Shin, C. Holland, and K. H. Lee, Biomacromolecules, 18, 2343 (2017).

26. K. Kataoka, and I. Uematsu, Kobunshi Ronbunshu, 34, 7 (1977).

27. Y. Takasu, Miscellaneous publication of the National Institute of Agrobiological Sciences, 7, 61 (2008).

28. S. Aonuma, K. Shindo, and S. Ihara, Sen-i Kikai Gakkaishi, 15, 321 (1962).

29. Y. Kawahara, T. Hananouchi, and H. Minami, J. Fiber Sci. Tech., 73, 328 (2017). 
30. Y. Magoshi, T. Hata, M. Kihara, K. Nagai, T. Tanaka, and J. Magoshi, J. Soc. Japanese Women Scientists, 11, 29 (2010).

31. https://www.jst.go.jp/kisoken/crest/report/sh_ heisei 10/shigen/magoshi.pdf.

32. J. Magoshi, and Y. Magoshi, Sen'I Gakkaishi, 63, P-244 (2007).

33. S. Ogiwara, “Kenshi no kozo"(T. Ito Ed.), Chikumakai, Ueda, pp. 20-45 (1957).

34. J. Magoshi, T. Tanaka, H. Sasaki, M. Kobayashi, Y. Magoshi, H. Tsuda, M. A. Becker, S. Inoue, and K. Ishimaru, Biomacromolecules, 4, 778 (2003).

35. S. Nakajima, Y. Kawahara, and E. Okonogi, J. Seric. Sci. Jpn., 62, 165 (1993).

36. R. E. Marsh, R. B. Corey, and L. Pauling, Biochem. Biophys. Acta, 16, 1 (1955).
37. B. Lotz, and H. D. Keith, J. Mol. Biol., 61, 201 (1971).

38. Y. Takahashi, M. Gehoh, and K. Yuzuriha, Int. J. Biol. Macromol., 24, 127 (1999).

39. R. E. Marsh, R. B. Corey, and L. Pauling, Acta Cryst., 8, 710 (1955).

40. M. Nagura, M. Urushidani, H. Shinohara, and H. Ishikawa, Koubunshi Ronbunshu, 35, 81 (1978).

41. P. C. J. Brunet, and B. C. Coles, Proc. Roy. Soc. Lond. $B, \mathbf{1 8 7}, 133$ (1974).

42. M. Yago, H. Sato, S. Hayashi, and A. Otsubo, Int. J. Wild Silkmoth \& Silk, 1, 203 (1994).

43. Y. Kawahara, M. Shioya, T. Kikutani, and A. Takaku, Sen'I Gakkaishi, 51, 1 (1995).

44. Y. Kawahara, and S. Nakajima, Sen'I Gakkaishi, 48, 671 (1992). 